\title{
“Energy levels and spectral lines of Ne VIII” [Eur. Phys. J. D 39, 173-188 (2006)]
}

\author{
K. Wilhelm ${ }^{1, a}$, W. Curdt ${ }^{1}$, I.E. Dammasch ${ }^{2}$, and D.M. Hassler ${ }^{3}$ \\ 1 Max-Planck-Institut für Sonnensystemforschung, 37191 Katlenburg-Lindau, Germany \\ 2 SIDC, Royal Observatory of Belgium, Circular Avenue 3, 1180 Uccle, Brussels, Belgium \\ 3 Southwest Research Institute, 1050 Walnut St., Suite 400, Boulder, CO 80302, USA
}

Received 8 February 2008

Published online 4 April 2008 - C) EDP Sciences, Società Italiana di Fisica, Springer-Verlag 2008

The authors of [1] provide a comprehensive compilation of Ne VIII energy levels and spectral lines. We are concerned about the data of one line treated in the second paragraph of Section 2, the line at $77.0 \mathrm{~nm}$. This line is very important for solar physics investigations as it is a bright line emitted in the lower corona of the Sun, where mass flux and energy transport determine the properties of the nascent solar wind. Consequently, it has been studied in a number of investigations, of which we want to mention two. The results are published by Peter and Judge [2] and Dammasch et al. [3]. Both these studies concluded that the rest wavelength of the Ne vIII line in question is $77.0428 \mathrm{~nm}$ with standard uncertainties of $0.7 \mathrm{pm}$ and $0.3 \mathrm{pm}$, respectively. Dammasch et al. discussed, in particular, the uncertainty level of $0.5 \mathrm{pm}$ stated by Bockasten et al. [4]. The conclusion was that it is far too optimistic and not reliable. So, we take issue with the statement of the authors that the Bockasten et al. measurements of this line are the most accurate in the literature.

If the Bockasten et al. value $(77.0409 \mathrm{~nm} \pm 0.0005 \mathrm{~nm})$ [4] for the rest wavelength of this line were true, it would imply downward movements in the solar corona that are in conflict with the measurements in other lines.

\section{References}

1. A.E. Kramida, M.-C. Buchet-Poulizac, Eur. Phys. J. D 39, 173 (2006)

2. H. Peter, P.G. Judge, Astrophys. J. 522, 1148 (1999)

3. I.E. Dammasch, K. Wilhelm, W. Curdt, D.M. Hassler, Astron. Astrophys. 346, 285 (1999)

4. K. Bockasten, R. Hallin, T.P. Hughes, Proc. Phys. Soc. 81, 522 (1963)

\footnotetext{
${ }^{a}$ e-mail: wilhelm@mps.mpg.de
} 